\title{
GEOMORPHOLOGY, CHARACTERISTICES AND CLASSIFICATION OF SOILS IN SOME AREAS IN ISMAILIA GOVERNORATE, EGYPT
}

\author{
M.S. Amira ${ }^{(1)}$, A.H. El-Nahry ${ }^{(2)}$, F.E. Abu Agwa ${ }^{(1)}$ and Shereen H. Ibrahim ${ }^{(1)}$ \\ (1) Soil Sci. Dept., Fac. Agric. Menoufia univ . \\ ${ }^{(2)}$ National Authority for Remote Sensing and Space Sciences (NARSS)
}

Received: Mar, 13, 2021

Accepted: Mar. 23, 2021

ABSTRACT: The current work aims to identifying the geomorphological, characteristics and classification of soils in some areas in Ismailia governorate, Egypt. The study area is located between longitudes $32^{\circ} 06^{\prime} 45^{\prime \prime}$ and $32^{\circ} 22^{\prime} 30^{\prime \prime} \mathrm{E}$ and latitudes 30 22' 30" and 30 57' 00" N. The integration of Remote Sensing (RS) and Geographic Information System (GIS) techniques was used to achieve this work.

The geomorphic map produced by processing and identifying the Landsat 8 image indicated that, the studied area has six main geomorphic units with different landforms. These units are: 1) Depressions, 2) Terraces (including Low, and High Terraces), 3) Basins (over flow basins and Decantation basins), 4) peneplains (Low and High), 5) Sandy plains (High, Moderate and Low) and 6) Mountain (Foot slope and Crest).

Twenty soil profiles were selected representing these units. The land and site features are observed and registered. The soil profiles were dug, morphologically described, and then samples were collected representing the subsequent layers in each profile for integrated physical and chemical analyses.

The studied area has almost flat with deep to very deep and well drained soils. Most of the studied soils have loamy sand texture and some parts have clay loam texture. The analytical data revealed that, the studied soils are slightly alkaline, mostly non-saline and haven't sodicity effect. The soils are moderately calcareous having Low gypsum and organic matter contents.

All studies soils haven't any diagnostic horizons, therefore they affiliated to Entisols and classified as Typic Torripsaments for $\mathbf{8 9 . 4 \%}$ and as Typic Torriorthents for $10.6 \%$ from the studied area.

Key words: RS, GIS, geomorphic units, landforms, soil characteristics, soil classification.

\section{INTRODUCTION}

The balance between the land and human resources is the most critical problem in Egypt. Accordingly, the major challenge of Egyptian government today is facing the need for better development and management of natural resources, to meet requirements of the fast-growing population. Therefore, horizontal expansion is considered the main focus for sustainable agricultural development in Egypt. Ministry of Agriculture and
Land Reclamation (MALR) formulated a strategy for sustainable agricultural development. One of the strategy objectives is to sustain the use of natural agricultural resources and to increase the productivity per units of both land and water. This strategy includes plans to cultivating about $\mathbf{1 . 5}$ million feddan in the year 2017 and increases to five million feddan by the year 2030 (FAO, 2013). One of the susceptible lands occurs mainly in the fringes of the Nile 
valley and Delta. Fringes of the Nile Delta are considered to be the most important location of the ambitious projects. These fringes have the most of best potentially suitable agricultural land resources for future expansion and development in Egypt (Noseir, 2014).

The identification

of geomorphological characteristics is from the important initial stages for studying any areas (Dawoud et al., 2005). Remote sensing (RS) is now recognized as an important tool in monitoring and managing natural resources (Lillesand and Kiefer, 2007). They added that, RS technique is one of the important methods that used for soil survey, mapping and environmental investigation. ESRI (2003) stated that, geographic information system (GIS) is a system for the management, analysis, and displaying geographic information, which is represented by a series of geographic datasets that model geography using simple, generic data structures.

The aim of the present work is to study the geomorphological, physiochemical characteristics and classification of the soils in some areas of Ismailia governorate. The integration of remote sensing (RS) and geographic information system (GIS) techniques was used perform all results and produce the spatial digital maps of this work.

\section{MATERIALS AND METHODS}

\section{Study area}

The study area occupies a part of Ismailia governorate, between longitudes

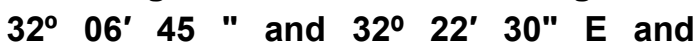
latitudes 30 22' 30" and 305 $57^{\prime} 00^{\prime \prime} \mathrm{N}$ as shown in Fig (1). Ismailia governorate is bordered from the north by Port Said governorate, from the east by North Sinai governorate, from the west by El-Sharkia governorate and from the south by Suez governorate. It covers an area of $\mathbf{1 0 1 4 . 2}$ $\mathrm{km}^{2}$ (241481.4 feddans). The studied area is characterized by a hot dry summer and warm winter with few rainfalls.

\section{Geomorphology of the study area}

The digital elevation model (DEM) of the study area was extracted from the Shuttle Radar Topography Mission (SRTM) and a topographic map with a scale of 1:25,000 covering the study area using Arc-GIS 10.7.1 software (ESRI, 2003). The Landsat 8 (path 178 / row 39) image acquired in 2018 and SRTM data were processed in ENVI 5.1 software (ITT, 2012) to identify the geomorphology and landforms of the studied area according to the approach developed by Dobos et al. (2002). The map legend was designed according to Zinck and Valenzuela (1990). ArcMap 10.7.1 software was used to display and produce geomorphic map of the study area with help of its DEM features and field observations (ESRI, 2014).

\section{Field work.}

Reconnaissance soil survey was conducted throughout the investigated area in order to acquire an appreciation of its broad soil patterns and characteristic landscape. The primary mapping units resulting from analysis of the DEM and interpretation information gained during unsupervised classification of Landsat images were verified.

Twenty soil profiles were chosen from three sample areas (Fig, 2) representing the geomorphic and landform units of the studied area. The soil profile's locations and elevations were defined in the field by using GPS system. The morphological description of these soil profiles was recorded on the basis outlined by FAO (2006). Soil samples were collected 
based on the vertical variations of each soil profile for the laboratory analyses of soil physical and chemical properties. Laboratory analyses

Particle size distribution, electrical conductivity (EC), pH, organic matter (OM), calcium carbonate $\left(\mathrm{CaCO}_{3}\right)$, gypsum contents, cation exchange capacity (CEC), exchangeable $\mathrm{Na}^{+}$ percentage (ESP) were determined according to Burt and Soil Survey Staff (2014). Identify class terms of each soil property was done according to Soil Science Division Staff (2017).

\section{Soil classification}

The soils of the studied area were classified up to sub great group level based on Soil Survey Staff (2014).

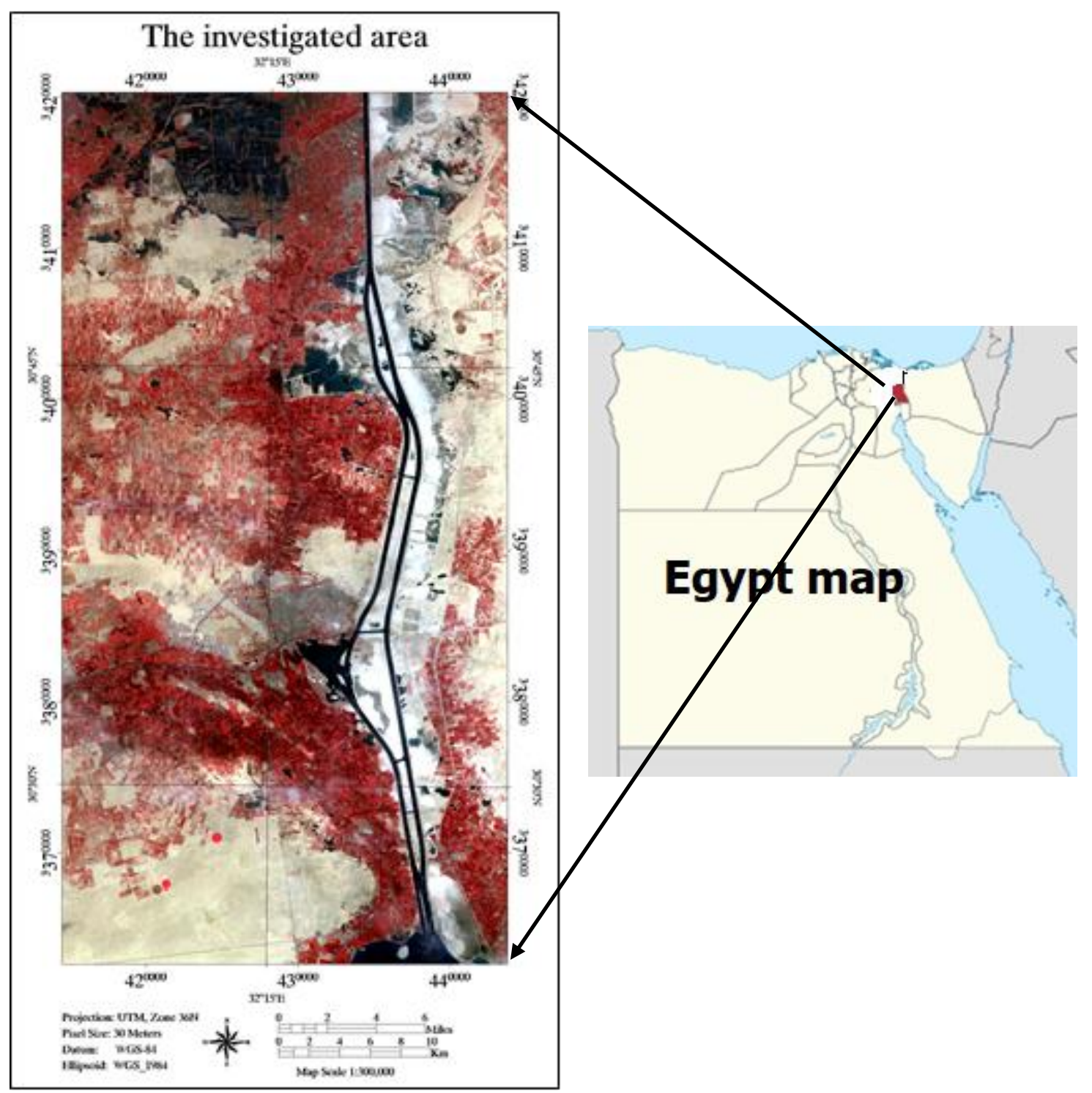

Fig (1): Study area. 


\section{Geomorphological map and profiles}

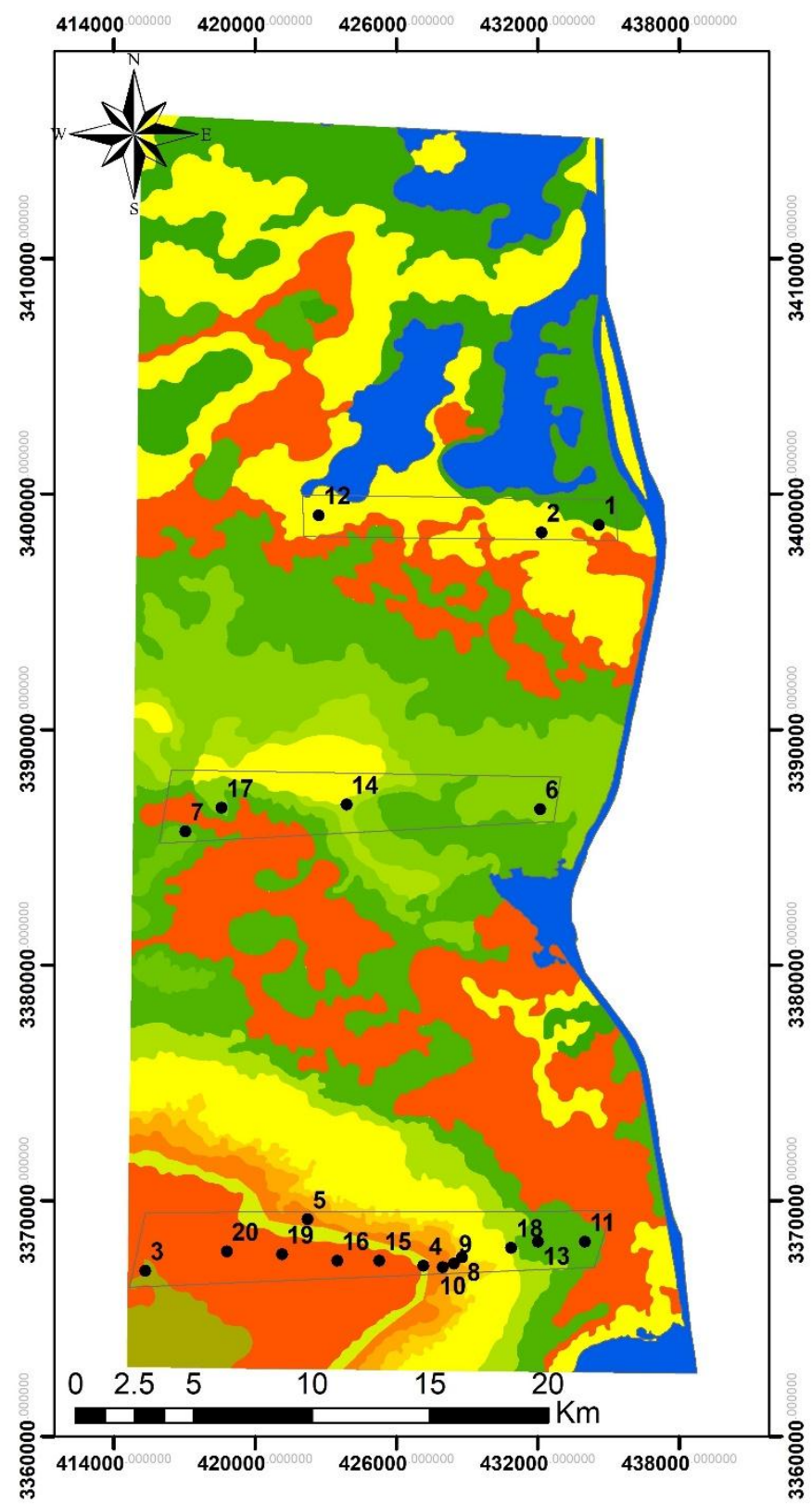

\section{Legend}

\begin{tabular}{|c|c|c|}
\hline \multirow{4}{*}{$\begin{array}{l}\text { profile } \\
\text { sample } \\
\text { area }\end{array}$} & Depressions & High Terraces \\
\hline & Decantation Basins & Peniplains \\
\hline & Over Flow Basins & High Peni plains \\
\hline & Low Terraces & Low Sandy Plains \\
\hline
\end{tabular}


Fig (2): Geomorphic units map and representative soil profile's locations of the study area.

\section{RESULTUS AND DISCUSSION Geomorphology}

Based on the satellite image treating, processing and interpretation with the aid of produced Digital Elevation Model (DEM), topographic maps and field observations, the integration of RS and GIS was used to identify the geomorphic and landform units of the study area. Produced DEM map of the study area presented in Fig (3) indicated that, the elevation for the whole of the study area is varied between $-4 \mathrm{~m}$ below sea level (b.s.l.) and increased gradually to $134 \mathrm{~m}$ above sea level (a.s.l.) at the south of the area.

The interpretation of satellite image of the study area indicated that, there are six main identified geomorphic units including seven subunits in this area. These units are 1) Depressions, 2) Terraces (Low and High), 3) Basins (over flow basins and Decantation basins), 4) Peneplains (Low and High), 5) Sandy plains (Low, Moderate and High) and 6) Mountain (foot slope and Crest). These units with their representative soil profiles (Fig, 2) and their areas are presented in Table (1). 
M.S. Amira, et al.,

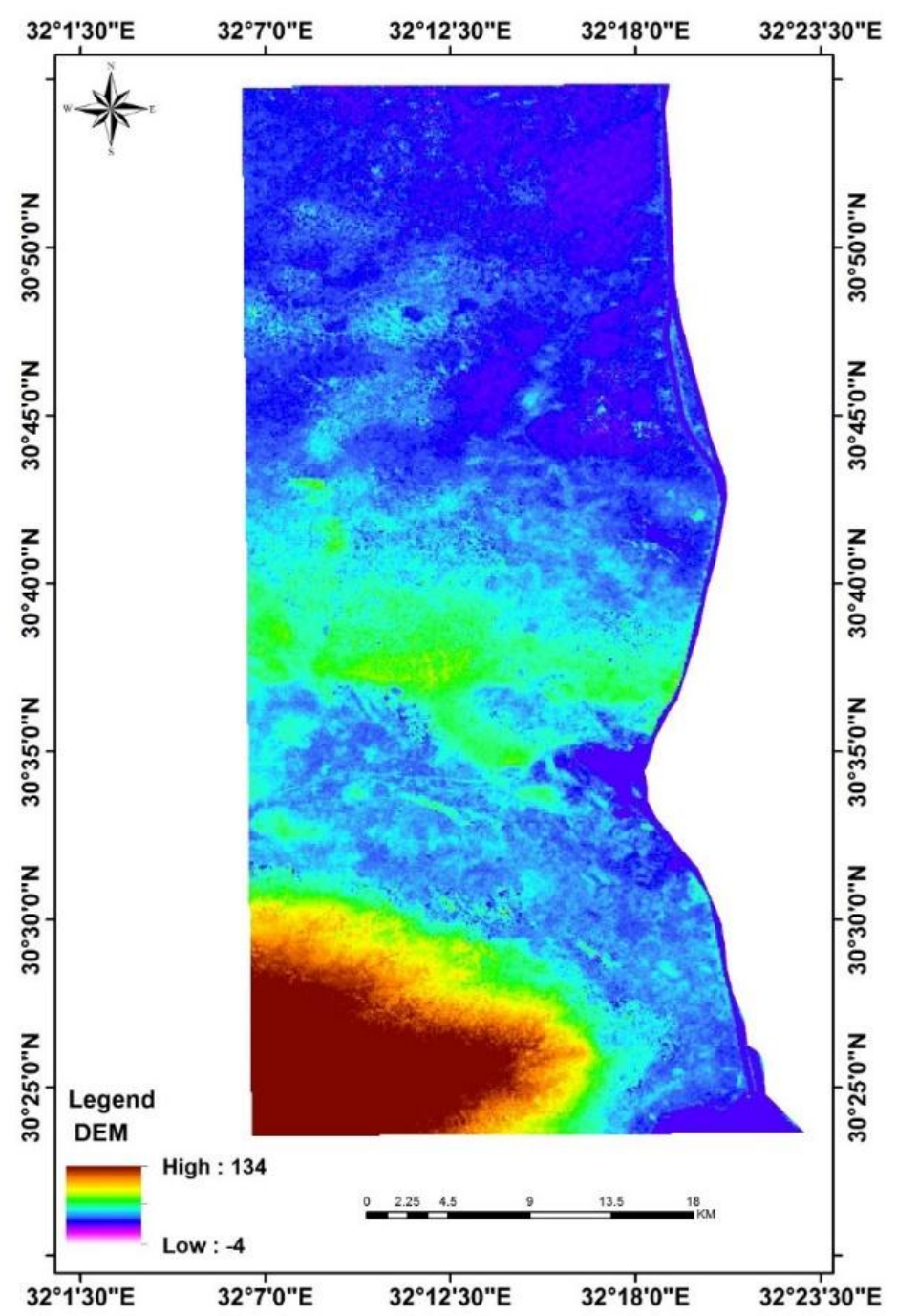

Fig (3): DEM map of the study area.

Table (1): Detailed geomorphic units of the study area.

\begin{tabular}{|c|c|c|c|c|c|c|c|}
\hline \multirow{2}{*}{$\begin{array}{c}\text { Geomorphic } \\
\text { units }\end{array}$} & \multirow{2}{*}{ Landform } & \multirow{2}{*}{$\begin{array}{l}\text { Mapping } \\
\text { Units }\end{array}$} & \multirow{2}{*}{$\begin{array}{c}\text { Profile } \\
\text { No }\end{array}$} & \multicolumn{3}{|c|}{ Area } & \multirow{2}{*}{$\begin{array}{c}\text { Total } \\
\%\end{array}$} \\
\hline & & & & $\mathrm{Km}^{2}$ & Fed & $\%$ & \\
\hline Depressions & Depressions & $\begin{array}{l}\mathbf{D} \\
\end{array}$ & 1 & 103.1 & 24541.9 & $10.16 \%$ & $10.16 \%$ \\
\hline \multirow{2}{*}{ Basins } & Decantation & BD & 11,13 & 196.0 & 46669.2 & $19.33 \%$ & \multirow{2}{*}{$20.08 \%$} \\
\hline & Over Flow & Bo & 7 & 7.6 & 1814.6 & $0.75 \%$ & \\
\hline \multirow{2}{*}{ Terraces } & High & Th & 14,18 & 96.2 & 22907.1 & $9.49 \%$ & \multirow{2}{*}{$13.86 \%$} \\
\hline & Low & TL & 6,17 & 44.3 & 10550.0 & $4.37 \%$ & \\
\hline \multirow{2}{*}{ Peneplains } & Low & $\mathbf{P}$ & 4 & 9.5 & 2259.6 & $0.94 \%$ & \multirow{2}{*}{$23.80 \%$} \\
\hline & High & PH & 2,12 & 231.9 & 55209.7 & $22.86 \%$ & \\
\hline \multirow{3}{*}{ Sandy Plains } & Low & SL & 8 & 11.0 & 2622.8 & $1.09 \%$ & \multirow{3}{*}{$3.70 \%$} \\
\hline & Moderately & SM & 9,10 & 14.4 & 3430.0 & $1.42 \%$ & \\
\hline & High & SH & 5 & 12.1 & 2879.9 & $1.19 \%$ & \\
\hline \multirow{2}{*}{ Mountain } & Foot slopes & MF & $15,16,19,20$ & 273.7 & 65157.3 & $26.98 \%$ & \multirow{2}{*}{$28.40 \%$} \\
\hline & Crest & ML & 3 & $\begin{array}{ll}14.4 \\
\end{array}$ & 3439.3 & $1.42 \%$ & \\
\hline \multicolumn{2}{|c|}{ Water bodies ${ }^{\star}$} & WB & & 107.1 & 25497.7 & \multicolumn{2}{|c|}{ Reference term } \\
\hline \multicolumn{4}{|c|}{ Tota } & 1014.2 & 241481.4 & $100 \%$ & $100 \%$ \\
\hline
\end{tabular}

* The different landforms areas \% are calculated considering the water bodies as reference term. 


\section{Soil morphology}

The morphological features of the studied soil profiles presented in Table (2) revealed that, the elevation of the studied soils is between $5 \mathrm{~m}$ to $112 \mathrm{~m}$ above sea level. The soils have almost flat topography with mostly deep and well-drained soil materials. The main hue notation of studied soil color is around brown notation (10YR). The soils have mostly weak to moderate medium to fine sub angular to granular structure with soft dry and friable consistence. The studied soils are moderately calcareous.

\section{Physiochemical properties}

The physiochemical properties of the studied soils are calculated as weighted profiles means (w.p.m.) and registered in Table (3). Data in this Table show that, most of the studied soils have loamy sand texture except few of them (profile, 4 and 11) that have sandy loam texture (w.p.m.). Most of the studied soils are non-saline to very slightly saline (EC $<2$ to $<4 \mathrm{dSm}^{-1}$; w.p.m.). Only soils of profile 15 in Mountain Foot Slope are slightly saline having $5.5 \mathrm{dSm}^{-1}$ (w.p.m.). All studied soils haven't sodicity effect and having slightly alkaline reaction having pH between 7.7 to 7.8 (w.p.m.). These soils are moderately calcareous having < Table (2): Morphological features of the studied soil profiles.

\begin{tabular}{|c|c|c|c|c|c|c|c|c|c|c|}
\hline \multirow{2}{*}{$\begin{array}{c}\text { Geomorphic } \\
\text { units }\end{array}$} & \multirow{2}{*}{$\begin{array}{l}\text { Land } \\
\text { forms }\end{array}$} & \multirow{2}{*}{$\begin{array}{c}\text { Profile } \\
\text { No. }\end{array}$} & \multirow{2}{*}{$\begin{array}{c}\text { Elevation } \\
\text { m asl }\end{array}$} & \multirow{2}{*}{$\begin{array}{c}\text { Depth } \\
\text { cm }\end{array}$} & \multicolumn{2}{|c|}{ Color } & \multirow{2}{*}{ Structure $^{1}$} & \multicolumn{2}{|c|}{ Consistence $^{2}$} & \multirow{2}{*}{ Boundary } \\
\hline & & & & & Dry & Moist & & Dry & Moist & \\
\hline \multicolumn{2}{|c|}{ Depression } & 1 & +8 & $\begin{array}{c}0-10 \\
10-50 \\
50-100\end{array}$ & $\begin{array}{l}\text { 10YR 5/1 } \\
\text { 10YR 6/6 } \\
\text { 10YR } 6 / 6\end{array}$ & \begin{tabular}{|l|}
$3 / 1$ \\
$5 / 4$ \\
$5 / 4$ \\
\end{tabular} & $\begin{array}{c}1 \mathrm{f} \text { sbk } \\
1 \mathrm{gr} \\
1 \mathrm{gr}\end{array}$ & $\begin{array}{c}\text { s hard } \\
\text { soft } \\
\text { soft }\end{array}$ & $\begin{array}{l}\text { v friable } \\
\text { v friable } \\
\text { v friable }\end{array}$ & $\begin{array}{c}\text { clear } \\
\text { diffuse } \\
-\end{array}$ \\
\hline \multirow{3}{*}{ Basins } & \multirow{2}{*}{ 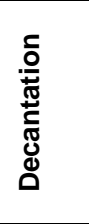 } & 11 & +11 & $\begin{array}{c}0-40 \\
40-60 \\
60-100\end{array}$ & $\begin{array}{l}\text { 10YR 5/2 } \\
\text { 10YR 4/2 } \\
\text { 10YR 4/2 }\end{array}$ & $\begin{array}{l}4 / 2 \\
3 / 2 \\
3 / 2\end{array}$ & $\begin{array}{c}1 \mathrm{~m} \text { to } \mathrm{f} \mathrm{sbk} \\
1 \mathrm{~m} \text { to } \mathrm{f} \mathrm{sbk} \\
2 \mathrm{gr}\end{array}$ & $\begin{array}{c}\text { s hard } \\
\text { soft } \\
\text { soft }\end{array}$ & $\begin{array}{c}\text { friable } \\
\text { v friable } \\
\text { v friable }\end{array}$ & $\begin{array}{c}\text { gradual s } \\
\text { diffuse } \\
\text { - }\end{array}$ \\
\hline & & 13 & +15 & $\begin{array}{c}0-30 \\
30-50 \\
50-70 \\
70-120 \\
\end{array}$ & $\begin{array}{l}\text { 10YR } 6 / 4 \\
\text { 10YR } 7 / 6 \\
\text { 10YR } 7 / 4 \\
\text { 10YR } 7 / 3 \\
\end{array}$ & $\begin{array}{l}5 / 4 \\
5 / 6 \\
6 / 4 \\
6 / 3 \\
\end{array}$ & $\begin{array}{c}1 \mathrm{~m} \text { to f sbk } \\
1 \mathrm{gr} \\
1 \mathrm{gr} \\
1 \mathrm{gr} \\
\end{array}$ & $\begin{array}{l}\text { soft } \\
\text { soft } \\
\text { soft } \\
\text { soft }\end{array}$ & $\begin{array}{c}\text { Friable } \\
\text { v friable } \\
\text { v friable } \\
\text { v friable }\end{array}$ & $\begin{array}{c}\text { clear } \\
\text { gradual s } \\
\text { gradual s } \\
- \\
\end{array}$ \\
\hline & ఏ’ & 7 & +17 & $\begin{array}{c}0-30 \\
30-50 \\
50-120\end{array}$ & $\begin{array}{l}\text { 10YR 6/4 } \\
\text { 10YR 6/6 } \\
\text { 10YR } 6 / 4\end{array}$ & $\begin{array}{l}5 / 4 \\
5 / 4 \\
5 / 4\end{array}$ & $\begin{array}{l}1 \mathrm{~m} \text { to } f \mathrm{sbk} \\
1 \mathrm{~m} \text { to } \mathrm{f} \mathrm{sbk} \\
1 \mathrm{~m} \text { to } \mathrm{f} \mathrm{sbk}\end{array}$ & $\begin{array}{c}\text { Soft } \\
\text { soft } \\
\text { s hard }\end{array}$ & $\begin{array}{c}\text { friable } \\
\text { v friable } \\
\text { s hard }\end{array}$ & $\begin{array}{c}\text { gradual s } \\
\text { clear } \\
-\end{array}$ \\
\hline Terraces & 3 & 6 & +13 & $\begin{array}{c}0-30 \\
30-80 \\
80-90 \\
90-120 \\
120-150\end{array}$ & $\begin{array}{l}\text { 10YR 5/6 } \\
\text { 10YR 5/6 } \\
\text { 10YR } 6 / 6 \\
\text { 10YR } 6 / 6 \\
\text { 10YR } 6 / 6\end{array}$ & $\begin{array}{l}4 / 6 \\
4 / 6 \\
5 / 6 \\
5 / 6 \\
5 / 6\end{array}$ & $\begin{array}{c}2 \mathrm{~m} \text { to } f \mathrm{sbk} \\
2 \mathrm{~m} \text { to } \mathrm{f} \mathrm{sbk} \\
1 \mathrm{~m} \text { to } \mathrm{f} \mathrm{sbk} \\
1 \mathrm{~m} \text { to } \mathrm{f} \mathrm{sbk} \\
1 \mathrm{f} \mathrm{sbk}\end{array}$ & $\begin{array}{c}\text { S hard } \\
\text { s hard } \\
\text { soft } \\
\text { soft } \\
\text { soft }\end{array}$ & $\begin{array}{l}\text { loose } \\
\text { loose } \\
\text { loose } \\
\text { loose } \\
\text { loose }\end{array}$ & $\begin{array}{l}\text { diffuse } \\
\text { diffuse } \\
\text { diffuse } \\
\text { diffuse } \\
-\end{array}$ \\
\hline
\end{tabular}

$7 \% \quad \mathrm{CaCO}_{3}$ content (w.p.m.). Gypsum content is very low (<1\%). Organic matter (OM) is very low (<1\%, as w.p.m.). The cation exchange capacity (CEC) is, also, low depending on the fine fractions and organic matter contents.

\section{Soil classification.}

Based on climatic condition, soil morphological, physiochemical characteristics the studied soils are classified up to sub great group level according to Soil Survey Staff (2014). According to FAO (1977) and USDANRCS (1997), the dominant soil moisture regime of this area is "Torric" with "Thermic" soil temperature regime. The studied area has mineral soils with Ochric Epipedon. All studied soils haven't any sub-surface diagnostic horizons. Therefore, they are affiliated to Entisols Order. Most of these soils (89.4\% from the study area) have about $80 \%$ or more sand fractions with sand to loamy sand texture and classified as Typic Torripsamments sub great group as presented in Table (4) and showed in Fig. (4). Only soils of profiles 4 in the Low Peneplains unit and 11 in the Decantation Basins (10.6\% from the study area have sandy loam texture (w.p.m) and classified as Typic Torriorthents. 
M.S. Amira, et al.,

\begin{tabular}{|c|c|c|c|c|c|c|c|c|c|c|}
\hline & & 17 & +15 & $\begin{array}{c}0-30 \\
30-50 \\
50-120\end{array}$ & $\begin{array}{l}\text { 10YR } 5 / 4 \\
\text { 10YR 6/4 } \\
\text { 10YR } 7 / 4\end{array}$ & $\begin{array}{l}4 / 4 \\
5 / 4 \\
6 / 4\end{array}$ & $\begin{array}{c}\mathrm{sg} \\
1 \mathrm{gr} \\
\mathrm{sg}\end{array}$ & $\begin{array}{l}\text { loose } \\
\text { soft } \\
\text { loose }\end{array}$ & $\begin{array}{c}\text { loose } \\
v \text { friable } \\
\text { loose }\end{array}$ & $\begin{array}{c}\text { gradual s } \\
\text { gradual s } \\
-\end{array}$ \\
\hline & 동 & 14 & +32 & $\begin{array}{c}0-30 \\
30-60 \\
60-90 \\
90-150\end{array}$ & $\begin{array}{l}\text { 10YR } 7 / 6 \\
\text { 10YR } 7 / 6 \\
\text { 10YR } 7 / 6 \\
\text { 10YR } 8 / 4\end{array}$ & $\begin{array}{l}6 / 6 \\
6 / 6 \\
6 / 6 \\
7 / 4\end{array}$ & $\begin{array}{l}1 \mathrm{gr} \\
1 \mathrm{gr} \\
1 \mathrm{gr} \\
1 \mathrm{gr}\end{array}$ & $\begin{array}{l}\text { soft } \\
\text { soft } \\
\text { soft } \\
\text { soft }\end{array}$ & $\begin{array}{l}\text { v friable } \\
v \text { friable } \\
v \text { friable } \\
v \text { friable }\end{array}$ & $\begin{array}{c}\text { diffuse } \\
\text { diffuse } \\
\text { diffuse } \\
-\end{array}$ \\
\hline & & 18 & +17 & $\begin{array}{c}0-30 \\
30-60 \\
60-120\end{array}$ & $\begin{array}{l}10 Y R \text { 4/4 } \\
\text { 10YR 6/4 } \\
\text { 10YR } 7 / 3\end{array}$ & $\begin{array}{l}3 / 3 \\
5 / 4 \\
5 / 3\end{array}$ & $\begin{array}{l}1 \text { f sbk } \\
1 \text { f sbk } \\
1 \text { f sbk }\end{array}$ & $\begin{array}{l}\text { soft } \\
\text { soft } \\
\text { soft }\end{array}$ & $\begin{array}{l}\text { v friable } \\
\text { v friable } \\
\text { v friable }\end{array}$ & $\begin{array}{c}\text { gradual s } \\
\text { gradual s } \\
-\end{array}$ \\
\hline \multirow{3}{*}{$\begin{array}{c}\text { Pen } \\
\text { plains }\end{array}$} & 3 & 4 & +8 & $\begin{array}{c}0-60 \\
60-100 \\
100-150\end{array}$ & $\begin{array}{l}10 Y R 5 / 3 \\
10 Y R 5 / 6 \\
\text { 10YR } 8 / 2\end{array}$ & $\begin{array}{l}5 / 2 \\
4 / 4 \\
6 / 2\end{array}$ & $\begin{array}{c}2 \mathrm{~m} \mathrm{sbk} \\
1 \mathrm{f} \mathrm{sbk} \\
\mathrm{sg}\end{array}$ & $\begin{array}{l}\text { hard } \\
\text { soft } \\
\text { loose }\end{array}$ & $\begin{array}{c}\text { friable } \\
\mathrm{v} \text { friable } \\
\text { loose }\end{array}$ & $\begin{array}{c}\text { abrupt } \\
\text { abrupt } \\
-\end{array}$ \\
\hline & \multirow[b]{2}{*}{ 高 } & 2 & +13 & $\begin{array}{c}0-70 \\
70-130\end{array}$ & $\begin{array}{l}\text { 10YR 6/4 } \\
\text { 10YR } 6 / 3\end{array}$ & $\begin{array}{l}5 / 4 \\
5 / 2\end{array}$ & $\begin{array}{l}1 \mathrm{~m} \text { to } f \mathrm{sbk} \\
1 \mathrm{~m} \text { to } \mathrm{sbbk}\end{array}$ & $\begin{array}{l}\text { soft } \\
\text { soft }\end{array}$ & $\begin{array}{l}\text { v friable } \\
\text { v friable }\end{array}$ & diffuse \\
\hline & & 12 & +10 & $\begin{array}{c}0-30 \\
30-50 \\
50-130\end{array}$ & $\begin{array}{l}\text { 10YR 5/2 } \\
\text { 10YR 6/3 } \\
\text { 10YR 4/2 }\end{array}$ & $\begin{array}{l}4 / 2 \\
5 / 3 \\
3 / 2\end{array}$ & $\begin{array}{l}1 \mathrm{~m} \text { to } f \mathrm{sbk} \\
1 \mathrm{~m} \text { to } \mathrm{fgr} \\
1 \mathrm{~m} \text { to } \mathrm{grr}\end{array}$ & $\begin{array}{c}\text { s hard } \\
\text { soft } \\
\text { soft }\end{array}$ & $\begin{array}{l}\text { Friable } \\
\text { v Friable } \\
\text { v friable }\end{array}$ & $\begin{array}{c}\text { abrupt } \\
\text { abrupt } \\
-\end{array}$ \\
\hline \multirow{4}{*}{ 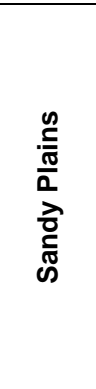 } & 3 & 8 & +5 & $\begin{array}{c}0-45 \\
45-65 \\
65-110 \\
110-140\end{array}$ & $\begin{array}{l}10 \text { YR } 5 / 8 \\
10 \text { YR } 5 / 8 \\
10 \text { YR } 5 / 6 \\
10 \text { YR } 6 / 8\end{array}$ & $\begin{array}{l}5 / 6 \\
5 / 6 \\
5 / 6 \\
5 / 6\end{array}$ & $\begin{array}{l}1 \mathrm{~m} \text { to } f \mathrm{sbk} \\
1 \mathrm{~m} \text { to } \mathrm{sbk} \\
1 \mathrm{~m} \text { to } f \mathrm{sbk} \\
1 \mathrm{~m} \text { to } \mathrm{sbk}\end{array}$ & $\begin{array}{l}\text { soft } \\
\text { soft } \\
\text { soft } \\
\text { soft }\end{array}$ & $\begin{array}{l}\text { v friable } \\
\mathbf{v} \text { friable } \\
\mathbf{v} \text { friable } \\
\mathbf{v} \text { friable }\end{array}$ & $\begin{array}{c}\text { diffuse } \\
\text { gradual s } \\
\text { gradual s } \\
-\end{array}$ \\
\hline & \multirow{2}{*}{$\begin{array}{l}\frac{\lambda}{\Phi} \\
\frac{1}{\pi} \\
\frac{0}{0} \\
\frac{0}{0} \\
\sum\end{array}$} & 9 & +27 & $\begin{array}{c}0-50 \\
50-120\end{array}$ & $\begin{array}{l}\text { 10YR 6/4 } \\
\text { 10YR } 6 / 6\end{array}$ & $\begin{array}{l}5 / 4 \\
5 / 6\end{array}$ & $\mid \begin{array}{c}2 \mathrm{~m} \mathrm{sbk} \text { to } g r \\
1 \mathrm{~m} \text { to } \mathrm{f} \mathrm{sbk}\end{array}$ & $\begin{array}{l}\text { soft } \\
\text { soft }\end{array}$ & $\begin{array}{l}\text { v friable } \\
\text { v friable }\end{array}$ & clear \\
\hline & & 10 & +19 & $\begin{array}{c}0-30 \\
30-80 \\
80-120\end{array}$ & $\begin{array}{l}\text { 10YR 4/2 } \\
\text { 10YR 6/3 } \\
\text { 10YR } 6 / 2\end{array}$ & $\begin{array}{l}3 / 2 \\
5 / 3 \\
5 / 2\end{array}$ & $\begin{array}{c}2 \mathrm{~m} \text { to } \mathrm{f} \mathrm{sbk} \\
1 \mathrm{~m} \text { to } \mathrm{f} \mathrm{gr} \\
1 \mathrm{~m} \text { to } \mathrm{f} \mathrm{gr}\end{array}$ & $\begin{array}{l}\text { s hard } \\
\text { soft } \\
\text { soft }\end{array}$ & $\begin{array}{c}\text { friable } \\
v \text { friable } \\
v \text { friable }\end{array}$ & $\begin{array}{c}\text { abrupt } \\
\text { gradual s } \\
-\end{array}$ \\
\hline & 동 & 5 & +49 & $\begin{array}{c}0-30 \\
30-50 \\
50-120\end{array}$ & $\begin{array}{l}10 Y R 5 / 4 \\
10 Y R 6 / 4 \\
10 Y R 6 / 6\end{array}$ & $\begin{array}{l}4 / 3 \\
5 / 3 \\
5 / 6\end{array}$ & $\begin{array}{c}1 \mathrm{f} \mathrm{sbk} \text { to } g r \\
1 \mathrm{~m} \text { to } \mathrm{fgr} \\
\mathrm{sg}\end{array}$ & $\begin{array}{l}\text { soft } \\
\text { soft } \\
\text { loose }\end{array}$ & $\begin{array}{c}\text { friable } \\
\text { v friable } \\
\text { loose }\end{array}$ & $\begin{array}{l}\text { clear } \\
\text { clear } \\
-\end{array}$ \\
\hline \multirow{5}{*}{ 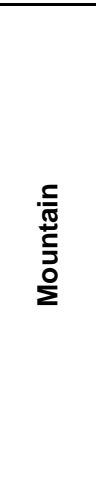 } & \multirow{4}{*}{ 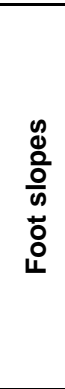 } & 15 & +78 & $\begin{array}{c}0-40 \\
40-60 \\
60-130\end{array}$ & $\begin{array}{l}\text { 10YR } 6 / 6 \\
\text { 10YR 6/4 } \\
\text { 10YR } 7 / 4\end{array}$ & $\begin{array}{l}5 / 4 \\
5 / 4 \\
6 / 4\end{array}$ & $\begin{array}{l}1 \mathrm{~m} \text { to } \mathrm{fgr} \\
1 \mathrm{~m} \text { to } \mathrm{fgr} \\
1 \mathrm{~m} \text { to } \mathrm{fgr}\end{array}$ & $\begin{array}{l}\text { soft } \\
\text { soft } \\
\text { soft }\end{array}$ & $\begin{array}{c}\mathbf{v} \text { friable } \\
\text { friable } \\
v \text { friable }\end{array}$ & $\begin{array}{c}\text { gradual s } \\
\text { gradual s } \\
-\end{array}$ \\
\hline & & 16 & +85 & $\begin{array}{c}0-30 \\
30-60 \\
60-150 \\
\end{array}$ & $\begin{array}{l}\text { 10YR } 6 / 6 \\
\text { 10YR } 7 / 4 \\
\text { 10YR } 6 / 4 \\
\end{array}$ & $\begin{array}{l}5 / 6 \\
6 / 4 \\
5 / 4 \\
\end{array}$ & $\begin{array}{l}1 \mathrm{~m} \text { to } \mathrm{fgr} \\
1 \mathrm{~m} \text { to } \mathrm{fgr} \\
1 \mathrm{~m} \text { to } \mathrm{fgr}\end{array}$ & $\begin{array}{l}\text { soft } \\
\text { soft } \\
\text { soft }\end{array}$ & $\begin{array}{l}\text { v friable } \\
v \text { friable } \\
\text { v friable } \\
\end{array}$ & $\begin{array}{c}\text { gradual s } \\
\text { gradual s } \\
-\end{array}$ \\
\hline & & 19 & +88 & $\begin{array}{c}0-30 \\
30-50 \\
50-120\end{array}$ & $\begin{array}{l}\text { 10YR } 6 / 6 \\
\text { 10YR } 6 / 4 \\
\text { 10YR } 6 / 4\end{array}$ & $\begin{array}{l}5 / 6 \\
5 / 4 \\
5 / 4\end{array}$ & $\begin{array}{l}1 \mathrm{~m} \text { to } \mathrm{fgr} \\
1 \mathrm{~m} \text { to } \mathrm{f} \mathrm{gr} \\
1 \mathrm{~m} \text { to } \mathrm{f} \mathrm{gr}\end{array}$ & $\begin{array}{l}\text { soft } \\
\text { soft } \\
\text { soft }\end{array}$ & $\begin{array}{l}\text { v friable } \\
\text { v friable } \\
\text { v friable }\end{array}$ & $\begin{array}{c}\text { gradual s } \\
\text { gradual s } \\
-\end{array}$ \\
\hline & & 20 & +81 & $\begin{array}{c}0-30 \\
30-60 \\
60-130\end{array}$ & $\begin{array}{l}\text { 10YR 5/3 } \\
\text { 10YR 6/4 } \\
\text { 10YR 6/4 }\end{array}$ & $\begin{array}{l}4 / 3 \\
5 / 4 \\
5 / 4\end{array}$ & $\begin{array}{l}1 \mathrm{~m} \text { to } \mathrm{f} \mathrm{gr} \\
1 \mathrm{~m} \text { to } \mathrm{f} \mathrm{gr} \\
1 \mathrm{~m} \text { to } \mathrm{f} \mathrm{gr}\end{array}$ & $\begin{array}{l}\text { soft } \\
\text { soft } \\
\text { soft }\end{array}$ & $\begin{array}{l}v \text { friable } \\
\text { v friable } \\
\text { v friable }\end{array}$ & $\begin{array}{c}\text { gradual s } \\
\text { gradual s } \\
-\end{array}$ \\
\hline & $\begin{array}{l}\bar{\phi} \\
\dot{\omega}\end{array}$ & 3 & +112 & $\begin{array}{c}0-25 \\
25-50 \\
50-85\end{array}$ & $\begin{array}{l}\text { 10YR 5/4 } \\
\text { 10YR 6/6 } \\
\text { 10YR 6/7 }\end{array}$ & $\begin{array}{l}4 / 3 \\
5 / 4 \\
5 / 4\end{array}$ & $\begin{array}{l}1 \mathrm{~m} \text { to } \mathrm{f} \mathrm{gr} \\
1 \mathrm{~m} \text { to } \mathrm{fgr} \\
1 \mathrm{~m} \text { to } \mathrm{f} \mathrm{gr}\end{array}$ & $\begin{array}{l}\text { soft } \\
\text { soft } \\
\text { soft }\end{array}$ & $\begin{array}{l}v \text { friable } \\
\text { v friable } \\
\text { v friable }\end{array}$ & $\begin{array}{c}\text { abrupt } \\
\text { clear } \\
-\end{array}$ \\
\hline
\end{tabular}

Abbreviations: Texture: Structure ${ }^{1}: 1=$ weak, 2 =moderate, $f=$ fine, $m=$ medium, gr = granular, sbk $=$ subangular blocky, $\mathrm{sg}=$ single grains; Consistence ${ }^{2}: \mathrm{v}=$ very, Boundary ${ }^{3}: \mathrm{s}=$ smooth

Table (3): Some physical and chemical properties of studied soil profiles.

\begin{tabular}{|c|c|c|c|c|c|c|c|c|c|c|c|c|c|c|}
\hline \multirow{2}{*}{ 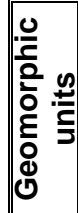 } & \multirow{2}{*}{ 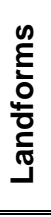 } & \multirow{2}{*}{ 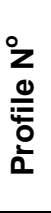 } & \multirow{2}{*}{$\begin{array}{l}\text { Depth } \\
\text { Cm }\end{array}$} & \multicolumn{3}{|c|}{$\begin{array}{c}\text { Particle size } \\
\text { distribution \% }\end{array}$} & \multirow{2}{*}{ 站 } & \multirow{2}{*}{ ִㅗㅇ } & \multirow{2}{*}{ 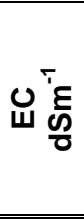 } & \multirow{2}{*}{ 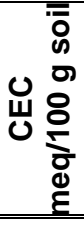 } & \multirow[b]{2}{*}{ ESP } & \multirow{2}{*}{ ర్ల } & \multirow{2}{*}{ 点 } & \multirow{2}{*}{$\begin{array}{l}\text { OM } \\
\mathrm{g} / \mathrm{kg}\end{array}$} \\
\hline & & & & 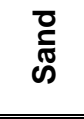 & 志 & $\frac{\vec{\pi}}{0}$ & & & & & & & & \\
\hline \multirow{4}{*}{ 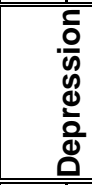 } & & \multirow{4}{*}{1} & $0-10$ & 63.6 & 22.8 & 13.6 & Sandy Loam & 7.7 & 1.0 & 16.2 & 6.8 & 5.7 & 0.17 & 0.11 \\
\hline & & & $10-50$ & 79.2 & 14.5 & 6.3 & Sandy Loam & 7.7 & 1.3 & 15.2 & 6.9 & 6.2 & 0.21 & 0.13 \\
\hline & & & $50-100$ & 84.5 & 10.9 & 4.6 & Loamy Sand & 7.7 & 1.2 & 15.0 & 8.9 & 6.5 & 0.23 & 0.13 \\
\hline & & & W.P.M & 80.3 & 13.5 & 6.2 & Loamy Sand & 7.7 & 1.2 & 15.2 & 7.9 & 6.3 & 0.22 & 0.13 \\
\hline \multirow{6}{*}{$\begin{array}{l}\stackrel{\mathscr{c}}{\frac{5}{D}} \\
\stackrel{\mathscr{D}}{\oplus}\end{array}$} & \multirow{6}{*}{ 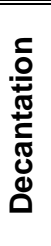 } & \multirow{4}{*}{11} & $0-40$ & 63.5 & 23.8 & 12.7 & \begin{tabular}{|l|} 
Sandy Loam \\
\end{tabular} & 7.7 & 1.5 & 11.3 & 7.6 & 5.8 & 0.12 & 0.23 \\
\hline & & & $40-60$ & 73.3 & 12.3 & 14.4 & Sandy Loam & 7.7 & 1.0 & 14.0 & 7.9 & 5.0 & 0.17 & 0.14 \\
\hline & & & $60-100$ & 85.5 & 10.9 & 3.6 & Loamy Sand & 7.8 & 1.2 & 12.5 & 8.3 & 5.9 & 0.11 & 0.28 \\
\hline & & & W.P.M & 74.3 & 16.3 & 9.4 & Sandy Loam & 7.7 & 1.3 & 12.3 & 7.9 & 5.7 & 0.13 & 0.23 \\
\hline & & \multirow{2}{*}{13} & $0-30$ & 84.0 & 11.5 & 4.5 & Loamy Sand & 7.7 & 1.3 & 15.0 & 10.4 & 5.5 & 0.12 & 0.16 \\
\hline & & & $30-50$ & 86.1 & 10.6 & 3.3 & Loamy Sand & 7.8 & 0.9 & 14.5 & 8.6 & 5.4 & 0.15 & 0.13 \\
\hline
\end{tabular}


Geomorphology, characteristices and classification of soils in some areas

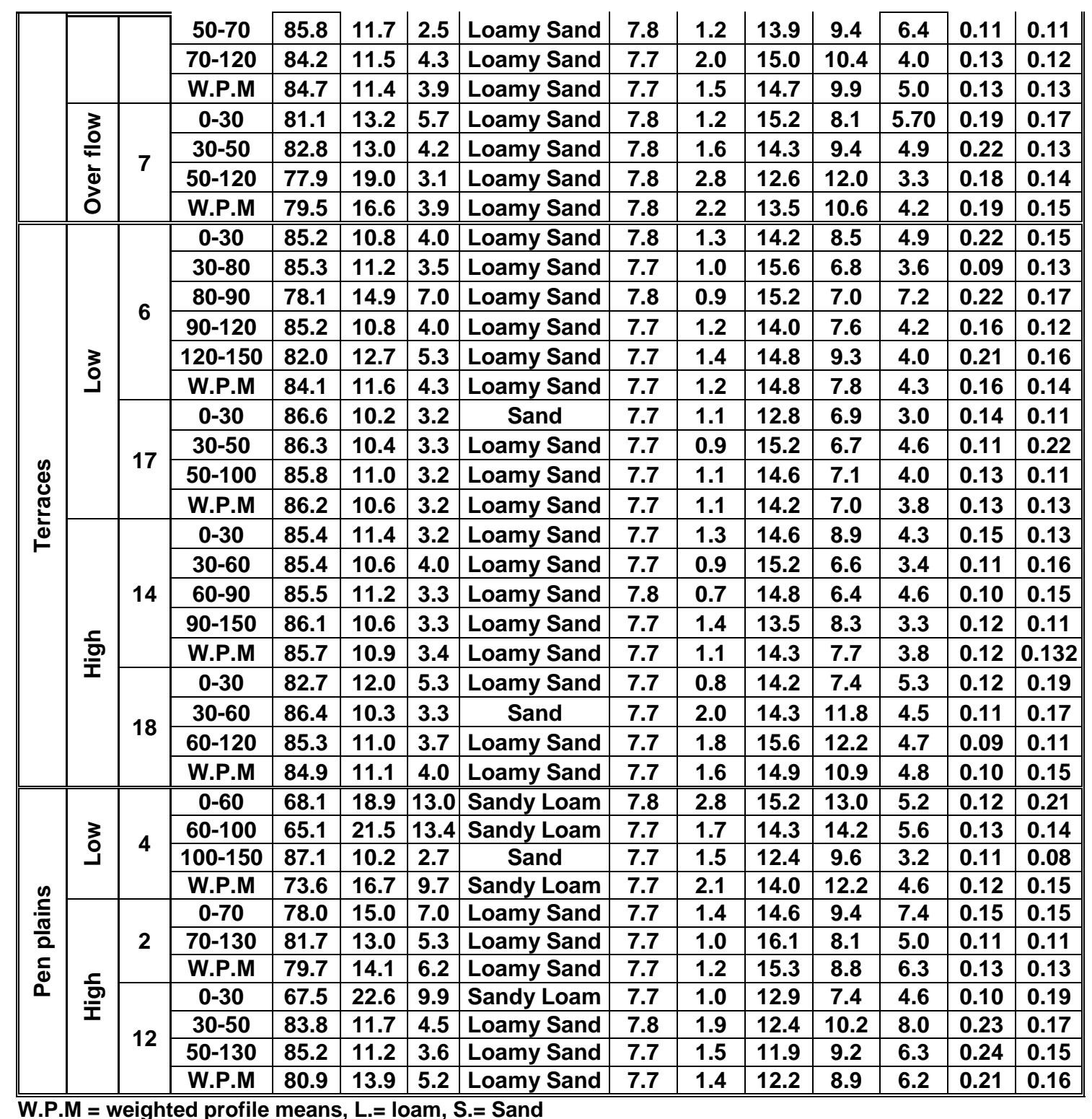

Table (3): Cont.

\begin{tabular}{|c|c|c|c|c|c|c|c|c|c|c|c|c|c|c|}
\hline \multirow{2}{*}{ 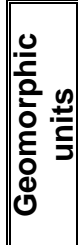 } & \multirow{2}{*}{ 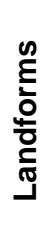 } & \multirow[b]{2}{*}{ 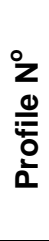 } & \multirow[b]{2}{*}{$\begin{array}{c}\text { Depth } \\
\text { Cm }\end{array}$} & \multicolumn{3}{|c|}{$\begin{array}{c}\text { Particle size } \\
\text { distribution \% }\end{array}$} & \multirow[b]{2}{*}{ 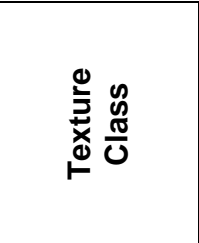 } & \multirow[b]{2}{*}{$=\frac{n}{2}$} & \multirow[b]{2}{*}{ } & \multirow{2}{*}{ 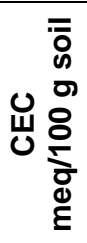 } & \multirow[b]{2}{*}{ ESP } & \multirow[b]{2}{*}{ ర్ల్ర } & \multirow[b]{2}{*}{ 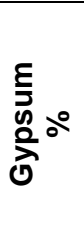 } & \multirow[b]{2}{*}{$\begin{array}{l}\text { OM } \\
\mathrm{g} / \mathrm{kg}\end{array}$} \\
\hline & & & & 을 & 志 & $\frac{\vec{\pi}}{0}$ & & & & & & & & \\
\hline \multirow{9}{*}{ 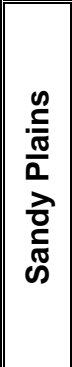 } & \multirow{5}{*}{ בَ } & \multirow{5}{*}{8} & $0-45$ & 80.8 & 13.2 & 6.0 & Loamy Sand & 7.7 & 2.2 & 13.5 & 12.9 & 4.3 & 0.23 & 0.15 \\
\hline & & & $45-65$ & 80.4 & 15.0 & 4.6 & Loamy Sand & 7.7 & 1.5 & 15.4 & 9.9 & 4.5 & 0.21 & 0.17 \\
\hline & & & $65-110$ & 80.1 & 14.9 & 5.0 & Loamy Sand & 7.8 & 1.7 & 14.2 & 10.0 & 5.3 & 0.27 & 0.16 \\
\hline & & & $110-140$ & 81.8 & 13.7 & 4.5 & Loamy Sand & 7.7 & 1.9 & 13.6 & 10.4 & 5.0 & 0.17 & 0.11 \\
\hline & & & W.P.M & 80.7 & 14.1 & 5.2 & Loamy Sand & 7.7 & 1.9 & 14.0 & 11.0 & 4.8 & 0.23 & 0.15 \\
\hline & \multirow{4}{*}{$\begin{array}{l}\frac{0}{\pi} \\
\frac{0}{0} \\
\frac{0}{0} \\
\Sigma\end{array}$} & \multirow{3}{*}{9} & $0-50$ & 81.3 & 13.5 & 5.2 & Loamy Sand & 7.7 & 1.2 & 12.4 & 8.2 & 5.2 & 0.19 & 0.13 \\
\hline & & & $50-120$ & 83.1 & 12.3 & 4.6 & Loamy Sand & 7.7 & 1.3 & 12.3 & 9.1 & 4.6 & 0.13 & 0.15 \\
\hline & & & W.P.M & 82.4 & 12.8 & 4.8 & Loamy Sand & 7.7 & 1.3 & 12.3 & 8.7 & 4.8 & 0.16 & 0.14 \\
\hline & & 10 & $0-30$ & 67.1 & 23.6 & 9.3 & Sandy Loam & 7.8 & 1.5 & 10.3 & 8.7 & 5.5 & 0.11 & 0.23 \\
\hline
\end{tabular}


M.S. Amira, et al.,

\begin{tabular}{|c|c|c|c|c|c|c|c|c|c|c|c|c|c|c|}
\hline & & & $30-80$ & 85.2 & 11.6 & 3.2 & Loamy Sand & 7.7 & 1.0 & 11.5 & 7.4 & 5.4 & 0.21 & 0.11 \\
\hline & & & $80-120$ & 81.8 & 12.8 & 5.4 & Loamy Sand & 7.7 & 1.2 & 15.2 & 8.2 & 5.7 & 0.23 & 0.13 \\
\hline & & & W.P.M & 79.5 & 15.0 & 5.5 & Loamy Sand & 7.7 & 1.2 & 12.4 & 8.0 & 5.5 & 0.19 & 0.15 \\
\hline & & & $0-30$ & 83.0 & 12.8 & 4.2 & Loamy Sand & 7.7 & 0.8 & 13.8 & 6.1 & 4.5 & 0.21 & 0.17 \\
\hline & 등 & & $30-50$ & 77.3 & 15.8 & 6.9 & Sandy Loam & 7.7 & 0.9 & 14.5 & 6.8 & 4.7 & 0.23 & 0.15 \\
\hline & 坣 & 5 & $50-120$ & 86.7 & 10.0 & 3.3 & Sand & 7.8 & 1.5 & 13.0 & 9.5 & 5.2 & 0.50 & 0.22 \\
\hline & & & W.P.M & 84.2 & 11.7 & 4.1 & Loamy Sand & 7.8 & 1.2 & 13.4 & 8.2 & 4.9 & 0.38 & 0.20 \\
\hline & & & $0-40$ & 80.8 & 13.9 & 5.3 & Loamy Sand & 7.7 & 8.3 & 13.5 & 13.0 & 5.0 & 0.11 & 0.12 \\
\hline & & 15 & $40-60$ & 85.2 & 10.8 & 4.0 & Loamy Sand & 7.7 & 7.3 & 14.2 & 12.2 & 4.2 & 0.13 & 0.11 \\
\hline & & 15 & $60-130$ & 85.8 & 10.5 & 3.7 & Loamy Sand & 7.7 & 3.3 & 15.4 & 6.3 & 4.3 & 0.11 & 0.14 \\
\hline & & & W.P.M & 84.2 & 11.6 & 4.2 & Loamy Sand & 7.7 & 5.5 & 14.6 & 9.3 & 4.5 & 0.11 & 0.13 \\
\hline & & & $0-30$ & 80.1 & 14.3 & 5.6 & Loamy Sand & 7.7 & 2.5 & 15.3 & 9.9 & 4.2 & .12 & 0.11 \\
\hline & & & $30-60$ & 85.8 & 11.0 & 3.2 & Loamy Sand & 7.7 & 1.9 & 16.2 & 9.9 & 3.6 & 0.13 & 0.13 \\
\hline & \& & 16 & $60-150$ & 85.3 & 10.3 & 4.4 & Loamy Sand & 7.7 & 1.9 & 15.3 & 9.1 & 3.2 & 0.11 & 0.09 \\
\hline & & & W.P.M & 84.4 & 11.2 & 4.4 & Loamy Sand & 7.7 & 2.0 & 15.5 & 9.4 & 3.5 & 0.12 & 0.10 \\
\hline & & & $0-30$ & 81.4 & 13.0 & 5.6 & Loamy Sand & 7.8 & 2.5 & 15.9 & 12.4 & 7.3 & 0.15 & 0.14 \\
\hline & 웅 & & $30-50$ & 82.6 & 12.6 & 4.8 & Loamy Sand & 7.7 & 2.3 & 12.3 & 10.9 & 5.0 & 0.11 & 0.12 \\
\hline$\stackrel{\mathbb{N}}{\underline{c}}$ & & 19 & $50-120$ & 85.8 & 11.0 & 3.2 & Loamy Sand & 7.8 & 1.7 & 16.5 & 10.1 & 4.6 & 0.15 & 0.13 \\
\hline & & & W.P.M & 84.1 & 11.8 & 4.1 & Loamy Sand & 7.8 & 2.0 & 15.6 & 10.8 & 5.3 & 0.14 & 0.13 \\
\hline & & & $0-30$ & 85.7 & 10.3 & 4.0 & Loamy Sand & 7.8 & 2.6 & 16.1 & 11.9 & 5.2 & 0.13 & 0.11 \\
\hline & & & $30-60$ & 85.8 & 11.2 & 3.0 & Loamy Sand & 7.8 & 2.4 & 14.2 & 10.6 & 4.0 & 0.12 & 0.13 \\
\hline & & 20 & $60-130$ & 82.3 & 13.6 & 4.1 & Loamy Sand & 7.7 & 2.0 & 15.3 & 9.7 & 4.3 & 0.11 & 0.14 \\
\hline & & & W.P.M & 83.9 & 12.3 & 3.8 & Loamy Sand & 7.7 & 2.2 & 15.2 & 10.4 & 4.4 & 0.12 & 0.13 \\
\hline & & & $0-25$ & 85.8 & 10.2 & 4.0 & Loamy Sand & 7.7 & 1.3 & 15.3 & 8.1 & 5.2 & 0.23 & 0.09 \\
\hline & & 3 & $25-50$ & 80.2 & 13.8 & 6.0 & Loamy Sand & 7.8 & 1.8 & 15.5 & 12.1 & 7.8 & 0.21 & 0.11 \\
\hline & பั & 3 & $50-85$ & 80.7 & 14.3 & 5.0 & Loamy Sand & 7.7 & 2.6 & 12.3 & 12.5 & 6.3 & 0.17 & 0.13 \\
\hline & & & W.P.M & 82.0 & 13.0 & 5.0 & Loamy Sand & 7.7 & 2.0 & 14.1 & 11.1 & 6.4 & 0.20 & 0.11 \\
\hline
\end{tabular}

Table (4): Soil classification in the study area.

\begin{tabular}{|c|c|c|c|}
\hline \multirow{2}{*}{ Order } & Sub Great Group & Soils of profiles No. & Area \\
\cline { 3 - 4 } Entisols & Typic Torripsamments & $\begin{array}{c}1,2,3,5,6,7,8,9,10,12, \\
13,14,15,16,17,18,19,20\end{array}$ & 89.4 \\
\cline { 2 - 4 } & Typic Torriorthents & 4,11 & 10.6 \\
\hline \multicolumn{2}{|c|}{ Total } & & 100 \\
\hline
\end{tabular}




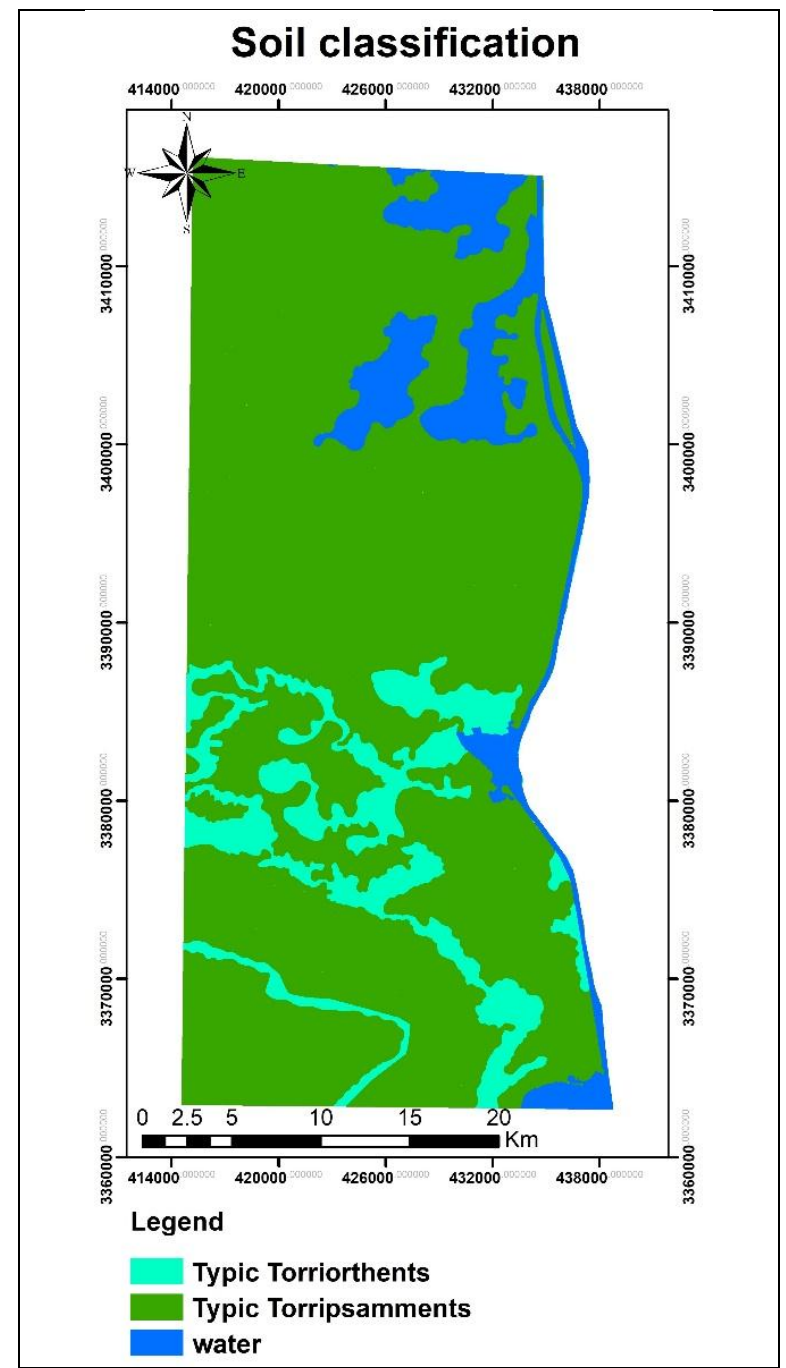

Fig (4): Spatial distribution of sub great groups in the study area.

\section{REFERANCES}

Burt, Rebecca and Soil Survey Staff (2014). Kellogg Soil Survey Laboratory Methods Manual, Soil Survey Investigations Report No. 42, Version 5.0, Kellogg Soil Survey Laboratory, National Soil Survey Center, Natural Resources Conservation Service, USDA, Lincoln, Nebraska, USA.

Dawoud, M.A., M.M. Darwish and M.M. ElKady (2005). GIS-based groundwater management model for Western Nile Delta. Water Resources. Manag. 19: 585-604.Dehaan, R, L. and Taylor, G, R. (2003): Image-derived spectral endmembers as indicators of salinization. International Journal of Remote Sensing, 24(4): 775-794.

Dobos, E., B. Norman, W. Bruee, M. Luca, J. Chris and M. Erika (2002). The use of DEM and satellite images for regional scale soil database. Proceedings of the 17th World Congress of Soil Science, Bangkok.

ESRI "Environmental Systems Research Institute" (2003): Using ArcGIS Geostatistical Analyst. Environmental Systems Research Institute (ESRI) Press, Redlands, California.

ESRI "Environmental Systems Research Institute" (2014): Arc Map Version 10.1 User Manual. ESRI, 380 New York 
M.S. Amira, et al.,

Street, Redlands, California, 923738100, USA.

FAO (1977). Soil map of the world $1: 5$ 000 000, Volume VI Africa, FAO, UNSCO, Paris.

FAO (2006). Guidelines for soil profile description. Soil Res. Dev. and Co. Serv., Land and Water Dev. Div., Rome, Italy.

FAO (2013). Country programing framework (CPF), Government of Egypt 2012 - 2017, The Ministry of Agriculture and Land Reclamation of the Government of Egypt and The Food and Agriculture Organization of the United Nations FAO, Rom, Italy.

Lillesand, T. M. and Kiefer R. W. (2007). Remote Sensing and Image Interpretation. 5th Ed. Paper back. John Wiley, New York.

Noseir, Dina M.A. (2014). Assessing the Potentials of Multi-functional Urban
Agriculture in Egypt, Towards Cultivating the New Urban Settlements "The Case of Al Sadat City", MSc. Thesis, Ain Shams Univ.

Soil Science Division Staff (2017). Soil Survey Manual, Handbook No. 1 USDA. 1400 Independence Avenue, SW, Washington, D.C. USA

Soil Survey Staff (2014). Keys to Soil Taxonomy, $11^{\text {th }}$ Ed., USDA, NRCS, Pocahontas Press, Inc., Blacksburg, Virginia, USA.

USDA-NRCS (1997). Soil climate map, Soil Science Division, World Soil Resources, United States Department of Agriculture \& Natural Recourses Conservation Service, Washington D.C.

Zinck, J.A. and C.R. Valenzuela (1990). Soil Geographic Database: Structure and Application Examples. ITC journal, 3: 270. 
جيومورفولوجيا وخصائص وتقسيم أراضي بعض مناطق محافظة الاسماعيلية، مصر

محمد سمير عراقي عميرة(1)، علاء الدين حسن النهري(2)، فوزي الثاذلي أبو عجوة(1)،

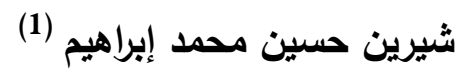

(1) قسم علوم الأراضي - كلية الززاعة - جامعة المنوفية

(2) الهيئة القومية للاستثعار من البعد وعلوم الفضاء - القاهرة

الملخص:

أجري هذا البحث بهدف دراسة الخصائص الجيومرفولوجية والفيزيوكيميائية وكنلك تقسيم أراضي بعض مناطق محافظة

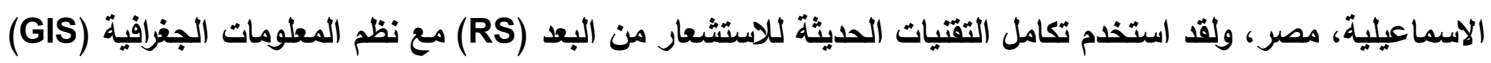

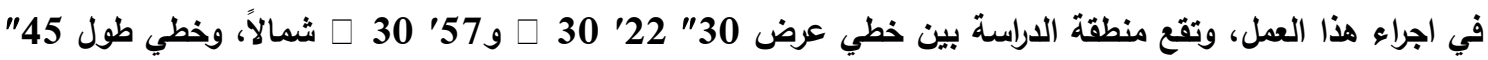

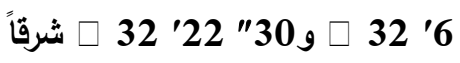

ولقد أوضحت الخريطة الجيومرفولوجية الناتجة من معالجة وتفسير الصور الجوية أن منطقة الدراسة تتميز بوجود

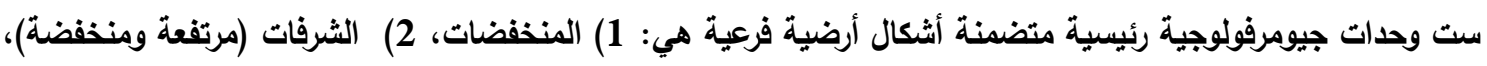

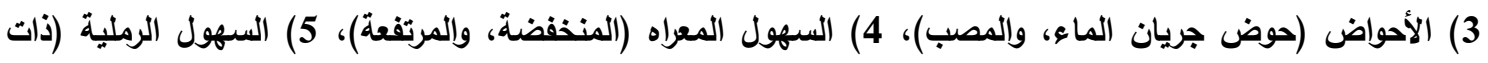

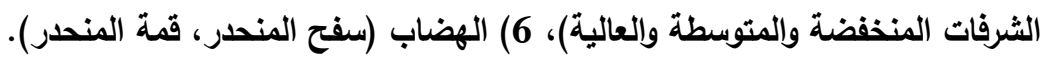

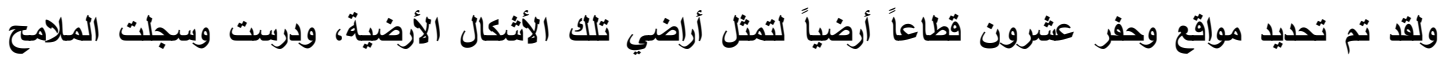

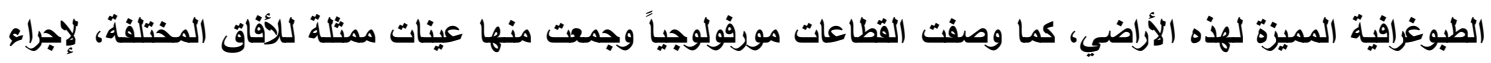
التحليلات المعملية لتقدير الخواص الطبيعية والكيماوية.

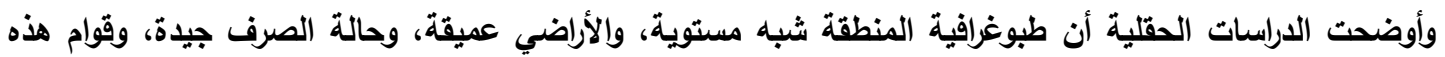
الأراضي يغلب عليه الطميي رملي ويعض الأراضي رملية طميية، ويناءها ضعيف من النوع الحبية الحبيهي.

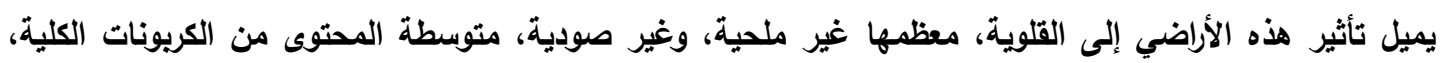
ونسبة الجبس والمادة العضوية ضئيلة، ويالتالي السعة التبادلية الكاتيونية منذفضة.

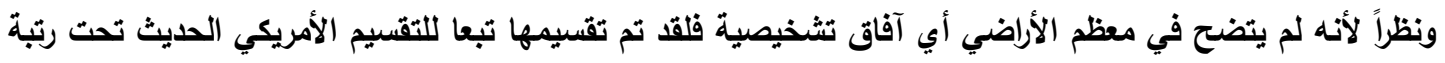

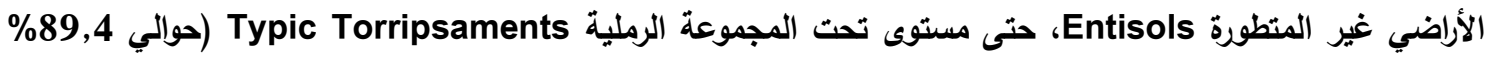

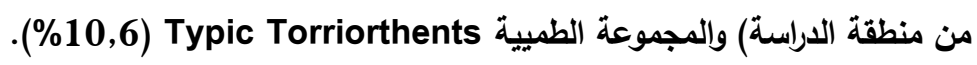

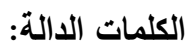
الاستثعار من البعد، نظم المعلومات الجغرافية، الوحدات الجيومورفولوجية، الخصائص الفيزيوكيميائية للأراضي، تقسيم الأراضي. 